\title{
8 Fazit
}

Die Forschung der letzten Jahrzehnte hat das frühe siebte Jahrhundert wiederholt als eine neuralgische Phase in der Entwicklung des östlichen Mittelmeerraums und des Nahen Ostens beschrieben. Vor diesem Hintergrund hat die vorliegende Arbeit den Blick auf die oströmische Monarchie gelenkt, auf die Möglichkeiten und Grenzen monarchischer Herrschaft. Die Regierungszeit des Herakleios - durch eine Vielfalt von Quellen dokumentiert - zeichnet sich in diesem Zusammenhang durch eine besondere Dynamik aus. Um die Funktionsweisen der oströmischen Monarchie in der Spätantike nachzuvollziehen, hat die Untersuchung sich auf zwei zentrale Faktoren konzentriert: das Verhältnis des Kaisers zur Hauptstadt und - damit in enger Verbindung stehend - das Verhältnis des Kaisers zum militärischen Sektor.

Grundlage dieser Arbeit war die Überzeugung, dass sich die Herrschaft des Herakleios nur dann in ihrer Entwicklungslogik beleuchten lässt, wenn man sie vor dem Hintergrund der hauptstädtischen Monarchie des 5. und 6. Jahrhundert interpretiert. In der Forschung wurde zuletzt wiederholt betont, dass die enge physische wie ideelle Bindung der oströmischen Monarchen an Konstantinopel ab dem frühen 5. Jahrhundert maßgeblich zur Sicherung von deren Position beitrug und damit - im Gegensatz zum Westteil des Reiches - das Fortbestehen des Kaisertums als Institution ermöglichte. In diesem Zusammenhang hat sich das Kapitel 2 zum Ziel gesetzt, die Beziehung zwischen den sesshaften Kaiser und dem Militär einer Revision zu unterziehen. Dabei wende ich mich gegen die These, dass der Rückzug des Kaisers aus dem aktiven Kriegsgeschäft eine Marginalisierung des militärischen Sektors zur Folge hatte. Im Gegensatz zu den vorigen Jahrhunderten scheint das Gros der in den Provinzen stationierten Soldaten bei der Aushandlung und Sicherung monarchischer Herrschaft tatsächlich keine zentrale Rolle mehr gespielt zu habe; obwohl ihnen das Privileg der Kaisernähe entzogen wurde, blieben sie auf den sesshaften Kaiser bezogen und ihm loyal, solange dieser ihre angemessene Versorgung sicherstellte. Anders verhielt es sich allerdings mit hohen militärischen Funktionsträgern - den Magistri militum, die qua Amt in den Rang der Illustres erhoben wurden und damit dem aktiven Konstantinopolitaner Senat angehörten - und auch mit den kaiserlichen Garden bzw. deren Kommandeuren. Ein Überblick über monarchische Sukzession ebenso wie Usurpation im 5. und 6. Jahrhundert hat gezeigt, dass in der Regel militärische Funktionsträger die ausschlaggebenden Impulse gaben. Die sesshaften Kaiser sahen sich den ständigen Versuchen der Einflussnahme hoher Militärs ausgesetzt. Dass die Jahrhunderte der hauptstädtischen Monarchie innenpolitisch verhältnismäßig stabil waren, lag darin begründet, dass die Kaiser ihrerseits Strategien entwickelten, mit denen sie die militärischen Funktionsträger zu einem gewissen Grad einzuhegen konnten: Zwischen Kaiser und hauptstädtischer Bevölkerung bildeten sich Interaktionsformen heraus, die auf der Sakralisierung des Kaisers einerseits und auf ostentativ zur Schau gestelltem Triumphalismus andererseits aufbauten - auf dem Umstand 
also, dass es den Kaisern als ewige Sieger gelang, die Erfolge der römischen Generäle, sofern es denn überhaupt Erfolge gab, auf sich zu beziehen.

Im frühen 7. Jahrhundert lässt sich eine Verschiebung des Verhältnisses zwischen Kaiser, Hauptstadt und militärischem Sektor nachvollziehen, die an zwei Phänomenen festzumachen ist: (1) 602 und 610 kam es zu gewaltsamen Machtwechseln in Konstantinopel, die dadurch ausgelöst wurden, dass römische Truppen bzw. Befehlshaber von außen Druck auf die Hauptstadt ausüben. (2) Herakleios, der sich 610 von Karthago aus an die Macht putschte, verließ als erster Kaiser nach über zweihundert Jahren Konstantinopel für mehrere Jahre am Stück, um die römischen Truppen persönlich in den Krieg zu führen. Die vorliegende Arbeit hat versucht herauszuarbeiten, warum es zu dieser Verschiebung kam. Anstatt von einer strukturellen Krise der hauptstädtischen Monarchie auszugehen, legt die Analyse in Kapitel 3 nahe, dass die Ereignisdynamiken zu einem gewissen Grad durch persönliches Versagen des Kaisers Maurikios bedingt waren; Maurikios schien sich der potentiellen Gefahr aus dem militärischen Sektor bewusst, war allerdings nicht in der Lage, angemessen darauf zu reagieren. Als entscheidender Einschnitt wurde der Moment identifiziert, in dem eine Revolte der Balkanarmee angesichts der unpopulären Direktiven des Kaisers derart eskalierte, dass auch in Konstantinopel Unruhen ausbrachen; anstatt sich der hauptstädtischen Bevölkerung zu stellen, floh Maurikios des Nachts nach Kleinasien. Aus diesem Machtvakuum heraus wurde Phokas, der Anführer der rebellierenden Truppen, zum Kaiser gekrönt. Dieses Ereignis, weder geplant noch vorhersehbar, eröffnete neue Handlungsspielräume, die sich dann nur wenige Jahre später die Herakleioi zu Nutze machten, deren Machtbasis in der militärischen Kontrolle Afrikas lag; sie lieferten die Probe aufs Exempel, dass militärischer Druck von außen die Machtverhältnisse in Konstantinopel wieder maßgeblich beeinflussen konnte. Ihr erklärtes Ziel, den regierenden Kaiser zu stürzen und Herakleios Junior an seiner Stelle zu installieren, konnte allerdings nur deswegen erreicht werden, da die hauptstädtische Bevölkerung sich gegen Phokas stellte, der die Akzeptanz, die ihm bei seiner Erhebung 602 entgegengebracht worden war, durch grobes Fehlverhalten systematisch verspielt hatte. Angesichts des Thronprätendenten, der mit einer Flotte im Hafen von Konstantinopel geankert hatte, entledigte die hauptstädtische Bevölkerung sich des Phokas und erhob stattdessen Herakleios zum Kaiser. Zum Ende des Kapitels wird anhand der Analyse der Krönungen von 602 und 610 nachvollzogen, wie externe Kandidaten zum Kaiser in Konstantinopel aufsteigen konnten: Durch ein auf die spezifischen Umstände angepasstes Ritual wurden Phokas und Herakleios in den hauptstädtischen Raum überführt und damit die Phase der akuten Desintegration performativ überwunden. Dass die Krönungen erstmals in Kirchen stattfanden - die des Phokas in der Johanneskirche am Hebdomon und die des Herakleios in der Hagia Sophie im Stadtzentrum - zeigt die wachsende Bedeutung des christlichen Referenzrahmens. Im Falle des Machtwechsels von 610 wird deutlich, dass bereits die Krönung des Herakleios mit der systematischen und äußerst wirkmächtigen Diffamierung des Phokas als Tyrann einherging, aus der heraus der neue Kaiser als gotterwählter Herrscher stilisiert werden konnte. Die Dynamiken der Jahre 602 und 610 
offenbaren, dass der Rückhalt der Hauptstadt weiterhin der ausschlaggebende Faktor für die Stabilität von Herrschaft war; das sollte sich auch in den Jahrzehnten darauf nicht ändern: Nur wer Konstantinopel hinter sich hatte, war Kaiser. Die spezielle Konfiguration von Monarchie blieb auf die Hauptstadt bezogen, mit dem Unterschied jedoch, dass nun der Kaiser nicht mehr in der Hauptstadt blieb.

Warum verließ Herakleios Konstantinopel über Jahre hinweg? Die Antwort auf diese Frage ist komplex und nur unter Berücksichtigung des Umstandes zu beantworten, dass die Perser sukzessive die Ostprovinzen des römischen Reiches besetzten. In Kapitel 4 habe ich allerdings dafür plädiert, dass auch interne Faktoren in Betracht gezogen werden müssen, um diesen Prozess zu erklären. Herakleios war darum bemüht, die kaiserliche Autorität über den militärischen Sektor wiederherstellen, zu deren Erosion er durch seine Usurpation selbst beigetragen hatte; es galt, dem Prozedere vorzubeugen, mit dem er selbst an die Macht gekommen war. Während Phokas noch an den Praktiken der sesshaften Monarchen festgehalten hatte - trotz seines militärischen Hintergrundes und des erneut entflammten Krieges gegen die Perser blieb er nach seiner Krönung in Konstantinopel - ging Herakleios neue Wege, indem er sich an die Spitze der römischen Truppen setzte. Die Entscheidung zur persönlichen Kriegsführung indes brachte eine Reihe schwerwiegender Risiken mit sich: nicht nur die Gefahr der Niederlage und eines damit einhergehenden Gesichtsverlustes, sondern auch den Umstand, dass der Kaiser damit die Verhaltenserwartungen des hauptstädtischen Umfeldes enttäuschte. Trotzdem brach Herakleios 624 auf eine wahre Himmelfahrtsmission auf, die ihn mehrfach bis ins persische Kernland brachte; zu diesem Zeitpunkt blieb ihm offenbar nur noch die Flucht nach vorne.

Herakleios' Versuch, den militärischen Sektor wieder enger an den Kaiser zu binden, ging mit Anstrengungen zur Sicherung seiner Position in Konstantinopel einher, die er vor allem in dem Bereich der Familienpolitik verfolgte. Durch die gezielte Förderung seiner Kernfamilie knüpfte der Kaiser ein engmaschiges dynastisches Netzwerk. 613, kurz bevor Herakleios erstmals als Oberbefehlshaber des Heeres nach Syrien aufbrach, krönte er seinen einjährigen Sohn Herakleios Konstantin zum Augustus. Trotz Herakleios' zeitweiliger Abwesenheit befand sich also immer ein Augustus in Konstantinopel, der, wenn auch minderjährig, zumindest die zeremonielle Funktion des Kaisers zu einem gewissen Grad ausfüllen konnte. Eine derart klare Designation beugte gleichzeitig auch Ambitionen militärischer Funktionsträger vor, die in der Vergangenheit regelmäßig versucht hatten, sich durch strategische Heiratsallianzen mit dem Kaiserhaus zu verbinden und so für die Nachfolge in Stellung zu bringen. Auch Herakleios' Entscheidung, sich in zweiter Ehe mit seiner Nichte Martina zu vermählen - eine nach römischem Recht illegitime Verbindung -, kann vor diesem Hintergrund als Maßnahme einer endogamen Familienpolitik interpretiert werden.

Was das frühe 7. Jahrhundert ausmacht, war - wie bereits gesagt - die Verschiebung im Verhältnis zwischen Kaiser, Hauptstadt und militärischem Sektor: Nach Jahrhunderten der sesshaften Monarchen stellte Herakleios' Abwesenheit von der Hauptstadt einen massiven Bruch mit etablierten Verhaltenserwartungen dar; das urbane Umfeld war die Präsenz des Kaisers gewohnt und forderte diese auch ein. Für 
diese Verschiebung musste nun eine Sprache gefunden werden; es musste ein Narrativ entwickelt werden, das Herakleios' Transgression gegenüber dem hauptstädtischen Umfeld vermitteln konnte. In Form der Gedichte des Georg von Pisidien sind uns Quellen erhalten, anhand derer sich dieser kommunikative Prozess im Detail nachvollziehen lässt: Mit seinen panegyrischen Epen, die als Gelegenheitsschriften auf rezente politische Entwicklungen reagierten, bildete Georg eine kommunikative Scharnierstelle zwischen (abwesendem) Kaiser, der Amtselite Konstantinopels und dem klerikalen Sektor - versammelt im zeremoniellen Interaktionsraum des Palastes. Das Kapitel 5 arbeitet heraus, dass die Sprache, die für den neuerlich kriegführenden Kaiser gefunden wurde, eine christliche war - eine Sprache, die sich von früheren Modellierungen des kriegführenden bzw. triumphierenden Kaisers abhob. Georg von Pisidien zeichnet Herakleios als strategos, als obersten Befehlshaber und betont damit die kaiserliche Autorität über das Militär; Herakleios’ persönliches Engagement auf dem Schlachtfeld indes, sein Feldherrentum, modelliert der Dichter entlang des Vorbilds Heiliger Männer und erschafft damit das Bild des titelgebenden schwitzenden Kaisers, der sich als Vorkämpfer des Christentums den zoroastrischen Persern entgegenstellt. Während Georg von Pisidien Herakleios nach dessen Sieg über die Perser vorm hauptstädtischen Publikum zum transzendenten Lichtwesen und Weltenerneuerer erhob, florierten in den rückgewonnenen Ostprovinzen akut eschatologische Interpretationen der rezenten Ereignisse.

Dass die Deutung des kriegführenden Kaisers christlich geprägt war, ist nicht per se verwunderlich, sondern reagierte auf gesamtgesellschaftliche Trends. Im frühen 7. Jahrhunderte lassen sich kaum mehr Ausdrucksformen finden, die nicht aus einem christlichen Referenzrahmen hervorgingen, bzw. in diesen eingepasst wurden. Es gab keine traditionelle, säkulare Geschichtsschreibung mehr, auch Theophylakt Simokattes schrieb seine Historien vor einem christlichen Deutungshorizont. Die Gesetzgebung, die noch unter Justinian ein zentrales, traditionsorientiertes Herrschaftsinstrument gewesen war und zugleich auch eine kaiserliche Repräsentationsform, lief beinahe vollkommen aus. In seiner gesamten Herrschaftszeit erließ Herakleios nur eine Hand voll Novellen, die sich noch dazu allesamt klerikalen Belangen widmeten. Vor diesem Hintergrund ist auch die viel diskutierte Änderung der kaiserlichen Titulatur unter Herakleios zu verstehen: aus dem Imperator Caesar Augustus wurde der - wohlgemerkt griechische - pistos en Christo basileus. Mischa Meier plädiert dafür, dass es unter Justinian zu eine Hypersakralisierung des Kaisers kam; er beobachtet eine hypertrophe Entrückung des Monarchen, der sich indes nicht mehr ausreichend von seinen Untertanen abheben konnte, die als Resultat einer gesamtgesellschaftlichen Liturgisierung als Volk von Heiligen auftrat. Davon ausgehend stellt Meier die These auf, dass kaiserliche Frömmigkeit als Maßnahme zur Herrschaftssicherung ab dem späten 6. Jahrhundert an Funktionalität einbüßte. Die intensive Analyse der Quellen des frühen 7. Jahrhundert legt dagegen nahe, dass sich Sakralisierung des Kaisers keineswegs totlief, dass Frömmigkeit stattdessen weiterhin als zentrale Kaisertugend angesehen und eingefordert wurde. Die Art und Weise jedoch, wie diese Frömmigkeit ausgedrückt und verhandelt wurde, passte sich an die neuen Gegeben- 
heiten an, nämlich daran, dass der Kaiser nun zumindest zeitweise aus dem hauptstädtischen Interaktionsnetz heraustrat. Dies fand schließlich auch in Herakleios' spektakulärer Restitutio Crucis im Jahr 630 einen Ausdruck - diskutiert in Kapitel 6 -, als Herakleios als erster christlicher Kaiser Jerusalem einen Besuch abstattete, um das Heilige Kreuz in die Anastasis-Kirche rückzuführen. Während die Forschung von der Annahme bestimmt wird, Herakleios habe sich mit der Rückführung des zentralen Kultobjektes explizit in Beziehung zum jüdischen König David gesetzt, zeigt die umsichtige Analyse der Evidenz, dass stattdessen andere Bezüge und Diskurse weit bedeutender waren. Die Hauptstadt etwa sah den Kaiser in seiner Sorge um das Heilige Kreuz als neuen Konstantin. Herakleios' persönliches Engagement für eine Einigung der christlichen Gemeinden im östlichen Mittelmeerraum, welches die Jahre nach der Restitutio Crucis bestimmte, diente konkret einer Konsolidierung der rückgewonnenen Ostprovinzen; gleichzeitig sind seine Bemühungen auf dem religionspolitischen Feld aber auch aus der Notwendigkeit heraus zu verstehen, sein kaiserliches Profil weiter zu schärfen: Während Herakleios sich in Syrien um eine Kompromissfindung bemühte, konnte Georg von Pisidien ihn in Konstantinopel als vom Heiligen Geist inspirierten Streiter für die Orthodoxie feiern, der nicht nur die Perser, sondern auch die Häresien in die Knie zwang.

Bemerkenswert sind in diesem Zusammenhang die Wechselwirkungen zwischen Kaiserbild und dem Selbstverständnis des hauptstädtischen Umfeldes: Die spezifische Deutung des kriegführenden Kaisers reagierte auf allgemeine gesellschaftliche Trends; aber gleichzeitig zeitigte monarchische Praxis ihrerseits Auswirkungen auf breitere gesellschaftliche Diskurse. Besonders gut lässt sich das in dem Moment nachvollziehen, als Konstantinopel 626 von Avaren und Persern belagert wurde und Herakleios weiterhin bei den Truppen in Ostanatolien weilte. Dass der Kaiser in diesem Moment seiner Fürsorgefunktion nicht nachkam, hätte politischen Konkurrenten eine Steilvorlage liefern können; zu einer Usurpation kam es jedoch gerade nicht. Stattdessen lässt sich an den Quellen nachvollziehen, wie die Kompetenz und Rolle des Kaisers auf alternative Akteure übertragen wurde, die nicht in politische Konkurrenz zum Monarchen standen, sondern dessen Position ergänzten, nämlich auf den Patriarchen Sergios und die Theotokos, deren Kult in Konstantinopel bereits hoch im Kurs stand. Diese Beobachtung legt folgenden Schluss nahe: Dass die Marienverehrung in Konstantinopel im frühen 7. Jahrhundert einen vorläufigen Höhepunkt erlebte, war eine Folge und Kompensation der langfristigen Abwesenheit des Kaisers von seiner Hauptstadt. Im frühen 7. Jahrhundert durchlief Konstantinopel eine Phase der städtischen Integration; die vorrangig religiös geprägten Sinnstrukturen, die sich unter Herakleios verfestigten und in denen auch dem Kaiser weiterhin eine zentrale Rolle zukam, förderten die Resilienz und Stabilität des hauptstädtischen Sozialgefüges. Dass das oströmische Reich an dem äußeren wie inneren Druck im 7. und 8. Jahrhundert nicht zugrunde ging, ist unter anderem eben diesem Umstand - dem Vorhandensein eines starken politischen und ideellen Zentrums - geschuldet, der einer endgültigen Fragmentierung des Reiches entgegenwirkte. 
Nach Jahren der politischen Instabilität gelang des Herakleios tatsächlich, seine Herrschaft vorübergehend zu sichern, die getroffenen Maßnahmen zeitigten den gewünschten Erfolg. Die arabische Expansion, die sein Restitutionswerk wieder zunichte machte, führte allerdings zu einer weiteren Dynamisierung seiner Herrschaft. Das Kapitel 6 blickt zuletzt auch auf die innenpolitischen Maßnahmen, mit denen Herakleios auf die vernichtende Niederlage der Römer am Yarmouk und den erneuten Verlust der Ostprovinzen reagierte. Nachdem sich in Konstantinopel eine Verschwörung gegen ihn aus der eigenen Familie zusammengebraut hatte, sah der Kaiser sich gezwungen, deutliche Zeichen zu setzen. Mit einer hyperthrophen Inszenierung einer Schiffsbrücke über den Bosporus, über die der Kaiser zu Pferd nach Konstantinopel einzog - versuchte er, den erlittenen Prestigeverlust zu kompensieren. Dass dieses spektakuläre Ereignis im Nachhinein einer satirischen Deutung unterworfen und als Maßnahme erinnert wurde, um die Wasserangst des Kaisers zu beschwichtigen, zeigt, dass triumphale Statements vor dem Hintergrund der katastrophalen Entwicklung im Osten nicht mehr wertgeschätzt wurden. Auch die öffentlichkeitswirksamen Maßnahmen, die Herakleios nach seiner Rückkehr in die Hauptstadt traf die Krönung eines weiteren Sohnes zum Augustus und die Promulgation der Ekthesis zur Schlichtung der monoenegetischen Kontroverse - konnten langfristig gesehen nicht die erhoffte Wirkung erzielen.

Als Herakleios im Jahr 641 eines natürlichen Todes starb, stand in Konstantinopel die Fortführung seiner Dynastie außer Frage; gleichzeitig offenbarten sich jedoch die Sollbruchstellen seiner Politik, wie das Kapitel 7 verdeutlicht. Die Förderung der Kernfamilie hatte zwar Herakleios' Position ebenso wie die seiner Söhne in Konstantinopel stärken können; der Umstand jedoch, dass es beim Tod des Dynastiegründers zwei Augusti in Konstantinopel gab (Herakleios Konstantin, Sohn aus erster Ehe mit Eudokia, und Heraklonas, Sohn aus zweiter Ehe mit Martina), führte zu unüberbrückbaren Spannungen und machte friedliche monarchische Sukzession geradezu unmöglich. Zwischen den beiden Familienlinien entbrannte ein erbitterter Machtkampf. Angesichts erneuter Instabilität in Konstantinopel geschah genau das, was Herakleios mit seiner Politik über Jahrzehnte recht erfolgreich zu verhindern gewusst hatte: Militärischen Funktionsträgern, die sich des Rückhaltes der Truppen in den Provinzen sicher waren, gelang es, Einfluss auf die Entscheidungsprozesse der Hauptstadt zu nehmen. Die innere Stabilität, die das oströmische Reich zwischen 610 und 641 ausgezeichnet hatte, war primär an Herakleios' Person gebunden gewesen; Krisenphänomene, die bereits im frühen 7. Jahrhundert zu beobachten gewesen waren, traten nach seinem Tod mit gesteigerter Vehemenz hervor: Unruhen von Seiten des Heeres und Usurpationsversuche von Militärs nahmen zu. Herakleios' Remilitarisierung des Kaisertums hatte neue Standards gesetzt: Dass der Kaiser sich wieder persönlich als Oberbefehlshaber engagierte, verlor recht schnell den transgressiven Charakter; der kollektive Erwartungshorizont in Hinblick auf kaiserliches Verhalten passte sich an. Die Augusti balancierten dabei jedoch auf einem schmalen Grat: Wie der Blick auf den Akzeptanzverlust und die Ermordung des Konstans II. im sizilischen Syrakus zeigt, musste die Abwesenheit des Kaisers von der Hauptstadt wohl be- 
gründet sein. Sowohl das hauptstädtische Umfeld als auch das Heer erwarteten ein militärisches Engagement im Sinne der Sicherung des Reiches, welches dem Druck der arabischen Expansion kaum standhalten konnte. Das dauerhafte Verweilen in einer alternativen Residenz, die Konstantinopels Monopolstellung Konkurrenz machte, dagegen wurde nicht toleriert.

Das frühe 7. Jahrhundert kann zurecht als eine neuralgische Phase in der Entwicklung der oströmischen Monarchie identifiziert werden - als Phase, während derer die politischen Strukturen, die die oströmische Monarchie des 5. und 6. Jahrhunderts geprägt hatten, zur Disposition standen, eine graduelle Umformung durchliefen und damit schließlich die Richtung für die weitere Entwicklung vorgaben. In den ersten Jahrzehnten des 7. Jahrhunderts erwiesen sich etablierte Handlungsoptionen in ihrer Verfügbarkeit bzw. Wirkmächtigkeit als unsicher, Normen und Routinen wurden in Frage gestellt, es kam zu Anpassungen von individuellen und kollektiven Erwartungshaltungen. Dabei ist allerdings zu betonen, dass die Monarchie des 7. Jahrhunderts sich weiterhin durch die grundlegenden Funktionsmechanismen auszeichnete, die sich - angelegt in der Einrichtung einer spezifisch römischen Form von Monarchie unter Augustus - über das 5. und 6. Jahrhundert hinweg im östlichen Mediterraneaum etabliert hatten: Der Mann auf dem Thron rang nach wie vor um Akzeptanz, seine Position war weiterhin prekär; das Zentrum des Reiches wie auch der Bezugspunkt kaiserlichen Handeln war und blieb Konstantinopel. Es ist also durchaus gerechtfertigt, diese Monarchie im 7. Jahrhundert weiterhin als eine römische, bzw. oströmische, zu qualifizieren und zu analysieren. Allein das Gewand dieses Herrschaftssystems - die Äußerungen und Sinnstrukturen, im Rahmen derer die Zeitgenossen ihre Umwelt wahrnahmen - hatte sich gegenüber dem Vergangenen grundlegend gewandelt; die Andersartigkeit dieses Gewandes ist es, was sich mit dem Begriff ,byzantinisch` bezeichnen lässt. 